\title{
Advancing Beyond Basal Insulin Therapy in Patients with Type 2 Diabetes
}

\author{
a report by \\ Craig W Spellman, PhD, DO
}

Assistant Dean, Professor of Medicine, Head of Endocrinology, and Director of the Diabetes Center, University of North Texas Health Science Center

DOI: 10.17925/USE.2007.00.1.34

An interesting report from the Quality of Care and Outcomes in Type 2 Diabetes (QuED) Study Group ${ }^{1}$ demonstrated that a physician's beliefs about glycemic control directly correlated with the degree of metabolic control achieved by his or her patients with type 2 diabetes (T2D). Only $14 \%$ of physicians targeted fasting plasma glucose (FPG) at $<110 \mathrm{mg} / \mathrm{dl}$ and about $40 \%$ thought that an FPG $>140 \mathrm{mg} / \mathrm{dl}$ was acceptable. Surprisingly, there was no difference between physicians in general practice and diabetologists with respect to the goals for fasting glucose. When patient performance was subsequently analyzed, average hemoglobin $A_{1 c}\left(A_{1 c}\right)$ was lowest among patients whose physicians emphasized tight glucose control, and $A_{1 c}$ remained at approximately $8 \%$ in patients managed by physicians with suboptimal expectations. It might be the case that the most significant barrier to early and aggressive management of $\mathrm{T} 2 \mathrm{D}$ is the healthcare professional.

People with T2D will eventually need insulin therapy because oral agents fail over time. Although we have data spanning decades demonstrating that tight control of glucose decreases end organ damage from diabetes, ${ }^{2-4}$ initiation of insulin therapy is often delayed for years. ${ }^{5}$ It is a common perception that insulin represents the last therapeutic measure and should be postponed for as long as possible. More recently, the Diabetes Attitudes, Wishes, and Needs (DAWN) study documented that there was much resistance to starting insulin therapy on the part of patients, nurses, and physicians. ${ }^{6}$ An analysis of National Health and Nutrition Examination Survey (NHANES) data by Koro et al. ${ }^{7}$ reported that only $36 \%$ of patients with T2D were treated to an $A_{1 c}$ goal of $<7 \%$. Further, only $27 \%$ of patients received insulin (16\% insulin only and $11 \%$ insulin plus oral agents). These attitudes about insulin must change if the current goals for glycemic control are going to be reached. The American Diabetes Association (ADA) recommends an $A_{1 c}$ of $<6 \%$ if possible without hypoglycemia. The American Association of Clinical Endocrinologists (AACE) and the American College of Endocrinology (ACE) recommend an $A_{1 c}$ of $<6.5 \%$; further, these goals should be reached within three to six months.

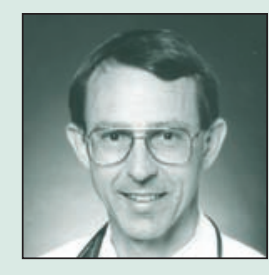

Craig W Spellman, PhD, DO, is Assistant Dean for the Dual Degree Programs in Medicine and Basic Science, Professor of Medicine, Head of Endocrinology, and Director of the Diabetes Center at the University of North Texas Health Science Center (UNTHSC) at Fort Worth, Texas. He is actively involved in basic research on the molecular mechanisms of peroxysome proliferator-activated (PPAR) agonists and the epidemiology of type 2 diabetes in children, and participates in multiple clinical trials at the UNTHSC. Dr Spellman is Chairman of the Medical Professionals and Health Care Professionals Advisory Committees for the Texas Diabetes Council.
Initial therapy for T2D usually begins with oral antidiabetic agents. Monotherapy is an option, but many clinicians now use dual therapy as the first intervention. The decision to start insulin is straightforward: Insulin is indicated as initial therapy if the presenting FPG is $>250 \mathrm{mg} / \mathrm{dl}$ and $A_{1 c}$ is $\geq 7.5 \%$ or if the patient has failed oral therapy, i.e. $A_{1 c} \geq 7.5 \%$. ${ }^{8}$ The latter cut-point derives from the observations that the effects of oral agents or exenatide are additive and that each additional agent will reduce $A_{1 c}$ only by about $0.5-1.5 \%$. Thus, if $A_{1 c}$ is $>7.5 \%$ on combination therapy, it is incumbent on the physician to begin insulin management.

Starting insulin therapy does not have to be an ordeal if presented properly to the patient. Much of the patient's angst about injections, hypoglycemia, weight gain, expense, and worsening diabetes can be dispelled by the physician. The doctor should broach insulin therapy at the first clinic visit. Further, an insulin injection with a modern pen device should be demonstrated at that first encounter. The healthcare provider needs to present accurately the benefits of insulin and the simplicity of modern insulin regimens. If this is not accomplished, patients will be reluctant to participate in achieving tight glycemic control.

Starting insulin can be easy. If the patient is motivated, a basal-bolus regimen can be implemented immediately. The total daily starting dose of insulin for the insulin-naïve patient is 0.25 units $/ \mathrm{kg}$ bodyweight. Half is given as glargine or detemir to cover basal glucose requirements and half is given as the bolus insulin with one of the fast analogs (glulisine, aspart, or lispro) divided between breakfast, lunch, and dinner to cover the prandial carbohydrate loads. The basal insulin is increased by two units every two days until the FPG is $<110 \mathrm{mg} / \mathrm{dl}$. The bolus insulin is increased by one to two units every two to three days for each meal until each two-hour postprandial glucose is $<140 \mathrm{mg} / \mathrm{dl}$ (ACE recommendation). Periodic reviews are needed to keep an approximate 50:50 ratio of basal to bolus insulin. ${ }^{9}$

However, not many patients are eager to jump directly to a basal-bolus regimen that entails four injections per day. Thus, another approach to starting insulin is to begin with 10 units of glargine or detemir once daily., The goal is to control the FPG to $<110 \mathrm{mg} / \mathrm{dl}$ to set the tone for the rest of the day. This is accomplished by adding two units of basal insulin every two days until the FPG target is reached. ${ }^{9}$ An alternative is to start with 10 units of pre-mixed insulin-such as 75\% neutral protamine lispro (NPL)/25\% lispro or $70 \%$ aspart protamine suspension (APS)/30\% aspart-once or twice daily ${ }^{8}$ and titrate as above..$^{9}$ Oral agents should be continued, ${ }^{10}$ although some clinicians discontinue a sulfonylurea when insulin is initiated. This approach appears simple at first glance, but it also becomes the root of the conundrum of how to advance insulin therapy. Oral agents plus basal 
insulin only may be insufficient to control post-prandial glucose. Bolus insulin is then needed. If a patient is already using enough basal insulin to achieve the FPG target-which may be 40-70 units/day-how then is bolus insulin added to cover the daytime glycemic excursions? How is the 50:50 ratio of basal to bolus insulin achieved? Does one decrease the basal dose? If so, what happens to FPG control? There is no codified consensus in response to these questions. ${ }^{8}$ Some physicians start the fast analog based on 'counting carbohydrates, ${ }^{8}$ while others arbitrarily give a fixed dose at each meal and then titrate. Experienced clinicians know that 'carbohyrdrate counting' is an excellent tool, but appreciate that it is too complex for most patients to understand or successfully implement. So, here is an approach to this problem using the arbitrary bolus method: Give $10 \%$ of the total daily dose as fast analog at the largest meal and decrease the basal insulin by $10 \%,{ }^{8}$ and titrate the bolus insulin to achieve a two-hour glucose of

Early and aggressive use of insulin is now the standard of care in type 2 diabetes, but only a minority of patients actually receive the benefits of modern insulin therapy.

$<140 \mathrm{mg} / \mathrm{dl}$. The same plan can then be extended to other meals to cover glycemic excursions. If the patient is using basal insulin only and the decision is made to advance to twice-daily pre-mix, then the transition involves dividing the total daily dose of basal insulin in half and giving one portion as pre-mix before breakfast and the other before dinner.

If a patient begins on a pre-mixed insulin schedule after failing oral therapy, control at certain time-points during the day will not be achieved because of the fixed proportions of long- and rapid-acting insulins. This will require a transition to basal-bolus therapy. One approach to advancing insulin management is to just start over and institute a basalbolus regimen. Because the patient is not insulin-naïve, the total daily dose can be 0.5 units $/ \mathrm{kg}$ bodyweight. Again, half is given as basal insulin and half is given as a fast analog divided between the meals. ${ }^{9}$ Another method is to give $80 \%$ of the total daily dose of the long-acting component of the pre-mix as basal insulin and then calculate $80 \%$ of the basal dose as the amount of fast analog to be divided between meals. For example, if a patient was using 30 units of 70/30 pre-mix twice a day (60 units/day), then the total dose of the long-acting component would be 42 units (i.e. $70 \% \times 60$ ). The dose of basal insulin then becomes about 34 units (i.e. $80 \% \times 42$ ). The dose of fast analog would be about 27 units (i.e. $80 \% \times 34$ ) divided between meals, or nine units before each meal. The utility of this approach is that in one step the patient is transitioned to basal-bolus therapy, time is saved by building on the prior titration, and the ratio of basal to bolus insulin will always be near optimum. ${ }^{9}$

With modern basal and bolus insulins, fasting and post-prandial glucose can be independently managed to obtain fine control. Consequently, the work of Monnier et al. ${ }^{11}$ has become an increasingly important tool in making therapeutic decisions on managing basal-bolus insulin therapy. Specifically, the group investigated the relationship between $A_{1 c}$ and various degrees of glycemic control. They concluded that $A_{1 c}$ values of $<7.3 \%$ correlated with postprandial glycemic excursions, while values of $>10 \%$ correlated with uncontrolled fasting hyperglycemia. The clinical applicability is apparent. If a patient's $A_{1 c}$ is in the $7 \%$ range, then emphasis should be placed on post-prandial glucose control. Glucose monitoring should capture two-hour post-prandial measurements, dietary education should be updated, and bolus insulin (not basal insulin) should be titrated.

\section{Concluding Remarks}

Early and aggressive use of insulin is now the standard of care in T2D, but only a minority of patients actually receive the benefits of modern insulin therapy. It appears that the healthcare provider may be the major barrier to timely changes in therapy. Patients may be resistant to starting insulin, but soon realize that the injection regimen is the trivial part of diabetes management. All approaches for initiating insulin are simple, while advancing therapy presents problems. Starting a basal-bolus schedule as the first insulin intervention ultimately requires the fewest therapeutic decisions; however, most patients do not enter an insulin pathway in this manner. Usually, basal insulin or a pre-mix is added to an oral regimen. Questions immediately arise when daytime glucose cannot be controlled and bolus coverage is needed. There is no universally accepted method and little basic research is available to bolster any particular approach for starting bolus therapy. However, the transition can be simplified, and the schemes outlined above have been used successfully in our clinics for the last six years. The diabetes educator now moves the patient forward from basal to basal-bolus therapy using the arbitrary bolus method. The diabetes educator advances from pre-mixed insulin management to basal-bolus therapy in one of two ways: If the patient is using fewer than about 40-60 units of pre-mix per day, then pre-mix is discontinued and basal-bolus therapy is started on a weight-adjusted basis ( 0.5 units $/ \mathrm{kg}$ bodyweight); if the patient had been using higher doses of pre-mix, then the ' $80 \%-80 \%$ ' rule is used because basal-bolus ratios are preserved and less time is lost titrating to an effective total daily insulin dose.
1. Belfiglio M, De Berardis $G$, Franciosi $M$, et al., The relationship between physicians self-reported target fasting blood glucose and metabolic control in type 2 diabetes, Diabetes Care, 2001;24: 423-9.

2. The Diabetes Control and Complications Research Group, The effect of intensive treatment of diabetes on the development and progression of long term complications in insulin-dependent diabetes mellitus, N Engl J Med, 1993;329:977-86.

3. Robertson KE, Prince MJ, What the UKPDS really says about cardiovascular disease and glycemic control, Clin Diabetes, 1999;17:109.

4. Nathan DM, Cleary PA, Bucklund JY, et al., Intensive diabetes treatment and cardiovascular disease in patients with type 1 diabetes, N Engl J Med, 2005;353:2643-53.

5. Brown JB, Nichole GA, Perry A, The burden of treatment failure in type 2 diabetes, Diabetes Care, 2004;27:1535-40.

6. Peyrot M, Rubin RR, Lauritzen T, et al., Resistance to insulin therapy among patients and providers: report of the cross-national Diabetes Attitudes, Wishes and Needs (DAWN) study, Diabetes Care, 2005:28:2673-9.

7. Koro CE, Bowlin SJ, Bourgeois N, Fedder DO, Glycemic control from 1988 to 2000 among U.S. adults diagnosed with type 2 diabetes: a preliminary report, Diabetes Care, 2004;27:17-20.

8. Hirsch IB, Bergenstal RM, Parkin CG, et al., A real-world approach to insulin therapy in primary care practice, Clinical Diabetes, 2005;23:78-86.

9. Texas Diabetes Council, available at: www.dshs.state.tx.us/diabetes/PDF/algorithms/INST2.pdf

10. Raskin $P$, Rojas $P$, Allen $E$, Comparison of twice-daily biphasic insulin 70/30 (BIAsp70/30) with once-daily insulin glargine (GLA) in patients with type 2 diabetes on oral antidiabetic agents, Diabetes, 2004;53(suppl. 2):602-P.

11. Monnier L, Lapinski $\mathrm{H}$, Colette $\mathrm{C}$, Contributions of fasting and postprandial plasma glucose increments to the overall diurnal hyperglycemia of type 2 diabetic patients: variations with increasing levels of HbA1c, Diabetes Care, 2003;26:881-5. 